\title{
Bifurcation analysis of a computer virus propagation model
}

\author{
Kenneth Dukuza(ii) \\ University of Pretoria, Department of Mathematics and Applied Mathematics, P/Bag X 20, Pretoria, \\ Republic of South Africa
}

\begin{abstract}
We propose a mathematical model for investigating the efficacy of Countermeasure Competing $(\mathrm{CMC})$ strategy which is a method for reducing the effect of computer virus attacks. Using the Centre Manifold Theory, we determine conditions under which a subcritical (backward) bifurcation occurs at Basic Reproduction Number $R_{0}=1$. In order to illustrate the theoretical findings, we construct a new Nonstandard Finite Difference Scheme (NSFD) that preserves the bifurcation property at $R_{0}=1$ among other dynamics of the continuous model. Earlier results given by Chen and Carley [The impact of countermeasure propagation on the prevalence of computer viruses, IEEE Trans. Syst., Man, Cybern. B. Cybern. 2004] show that the CMC strategy is effective when the countermeasure propagation rate is higher than the virus spreading rate. Our results reveal that even if this condition is not met, the CMC strategy may still successfully eradicate computer viruses depending on the extent of its effectiveness.
\end{abstract}

Mathematics Subject Classification (2020). 65L05, 93A30, 93D20

Keywords. basic reproduction number, bifurcation, computer virus, countermeasure competing, nonstandard finite difference scheme

\section{Introduction}

Electronic communications media have become major role players in our daily lives because of their efficiency in exchanging information, processing transactions, capturing biometric data etc, all of these can be done in real time. However, as impressive as all this sounds, it comes at an enormous cost in the event of a serious cyber attack such as denial of service, unauthorised access, financial fraud, theft or even loss of proprietary information from mobile devices and other vulnerable sources, theft or loss of customer data, malware etc; see [7].

Several organisations minimise the risk of these attacks by putting in place a combination of countermeasure strategies such as antivirus and antispyware software, application level firewalls, encryption of data, vulnerability and patch management tools, web or URL filtering etc. The report [7] suggests that all these efforts are still far from being adequate, and thus cyber security remains a cause for concern.

In this paper we develop a mathematical model to further investigate the impact of

Email address: kenneth.dukuza@up.ac.za

Received: 07.06.2020; Accepted: 26.04.2021 
CMC strategy on the spread of computer viruses. The CMC strategy assumes, among other things, that the propagation rates of countermeasures and viruses are different; see [6] for a detailed description. Inspired by the models found in mathematical epidemiology and the striking analogy between human and computer virus attacks [19], we formulate a compartmental model; see for instance [4]. This approach is not new, refer to $[13,18,20,23,24]$ etc. Our approach is motivated by the fact that epidemiological models have a disease progression or nonprogression threshold which is known as the basic reproduction number $[13,15,16,21]$, and is usually denoted by $R_{0}>0$. In general if $R_{0}<1$, then the disease dies out in the long run, whereas if $R_{0}>1$ then the disease becomes endemic in the population. On the other hand, if the model undergoes a backward bifurcation at $R_{0}=1$, the disease might persist even when $R_{0}<1$. In order to control the disease in the latter case, $R_{0}$ has to be reduced until it is much smaller than one.

In a mathematical model by [24], four potential equilibria were determined and analysed. Their results suggested that $\mathrm{CMC}$ is effective, but did not make any mention of the degree of efficacy of the countermeasure strategy. On the other hand, in [5] the authors were more specific in their findings that the CMC strategy reduces the scale of virus attack significantly when the countermeasure propagation rate is higher than the virus spreading rate.

It is worth mentioning that, in mathematical epidemiology, the term 'backward bifurcation phenomenon' is very prominent, and is understood to refer to the region whereby a stable disease-free equilibrium, a small endemic equilibrium, and a large endemic equilibrium co-exist while $R_{0}<1$. This terminology is mostly found in scholarly articles, see for instance [5] and [9], and seldomly in standard texts of dynamical systems. In the interest of local analysis and the Centre Manifold Theory upon which the main Theorem in [6] is predicated, we will use the following nomenclature: subcritical(backward) and supercritical(forward) bifurcations instead. The main objectives of this paper are as follows:

(i) To show that even in a case whereby the virus spreading rate is higher than the countermeasure propagation rate, the virus can still be eradicated if the countermeasures are effective enough (i.e. they suppress the infection rate adequately).

(ii) To construct a new NSFD scheme which preserves the bifurcation property at $R_{0}=1$.

(iii) To illustrate our results using numerical simulations.

By using the Centre Manifold Theory [5], [22], we will prove the existence of a subcritical bifurcation whereby a disease might persist even though $R_{0}<1$ and make recommendations thereafter.

The rest of the paper is organised as follows: Section 2 deals with formulation of the model, while model analysis is done in Section 3. Bifurcation analysis is found in Section 4. Section 5 is devoted to numerical simulations and discussion. We conclude this work in Section 6.

\section{Formulation of the model}

Without causing any confusion among computer scientists and others in related fields, we will use the term virus for all malware. "Internal computers for computers already connected in the internet network, External computers for computers not connected to the internet network". The model state variables are given in Table 1 while Table 2 displays parameters.

\subsection{The model}

Model (2.1) looks very similar to the model in [24] at first glance, but their dynamics are quite different. In our model the assumption that computers with countermeasures may be infected by the virus without becoming susceptible first is key. In most cases, 
Table 1. State variables

\begin{tabular}{c|l}
\hline State & Number of Computers at time $t$ \\
\hline Susceptible: $\mathrm{S}(\mathrm{t})$ & $\begin{array}{l}\text { that have no countermeasures at all } \\
\text { (i.e. a set of external computers connecting to the network } \\
\text { for the first time, together with internal computers } \\
\text { that have been infected and cured). }\end{array}$ \\
Countermeasured: $\mathrm{C}(\mathrm{t})$ & $\begin{array}{l}\text { Infious: } \mathrm{I}(\mathrm{t}) \\
\text { that have acquired the virus and are capable } \\
\text { of infecting other computers in the network. } \\
\text { acquired countermeasures, and are also } \\
\text { capable of disseminating or propagating } \\
\text { countermeasures to all the internal computers } \\
\text { (i.e. both S and I). These computers have much reduced } \\
\text { measure of vulnerabilty to infection due to acquired } \\
\text { countermeasures. }\end{array}$ \\
\hline
\end{tabular}

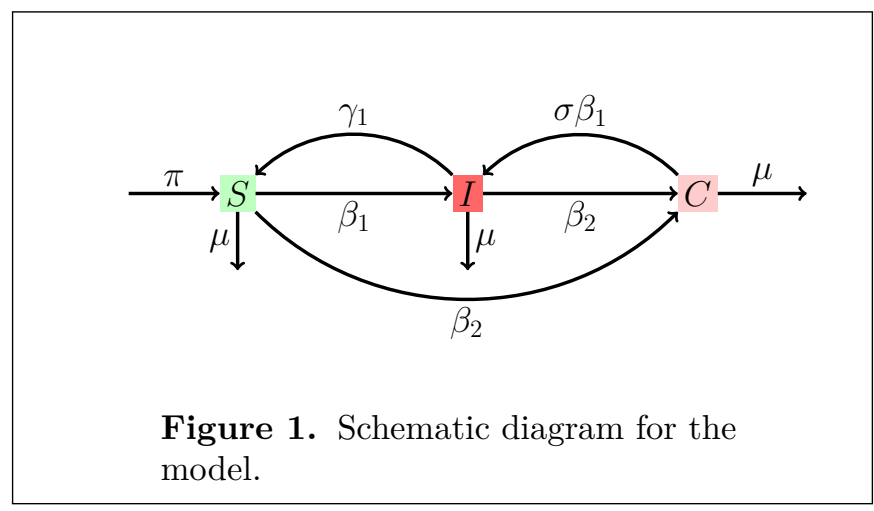

computers with countermeasures are usually considered to be relatively safe from virus attacks and receive little attention until such time as they show symptoms of infection. The fraction $\sigma$ of computers that resist the virus attack is very critical in the efficacy of the CMC strategy, and this will be elucidated in the results of this paper. External computers get connected to the network through the $S$ class at the rate $\pi$ and are all susceptible to the virus attack. Soon thereafter, they are equipped with some form of antivirus and proceed to the $C$ class at the rate $\beta_{2}$. The computers in class $C$ are also capable of disseminating countermeasures to $I$ and $S$ at the rate $\beta_{2}$. The infected computers $I$ are patched and fixed at the rate $\gamma_{1}$ and join the $S$ class, and remain there until they receive countermeasures again.

$$
\left\{\begin{aligned}
\dot{S} & =\pi-\beta_{1} S I-\beta_{2} S C+\gamma_{1} I-\mu S, \\
\dot{I} & =\beta_{1} S I-\beta_{2} C I-\left(\gamma_{1}+\mu\right) I+\sigma \beta_{1} C I, \\
\dot{C} & =\beta_{2} C I+\beta_{2} S C-\sigma \beta_{1} C I-\mu C,
\end{aligned}\right.
$$

where $\{S(0), I(0), C(0)\} \in \mathbb{R}_{+}^{3}$.

\section{Model analysis}

Let

$$
N(t)=S(t)+I(t)+C(t)
$$


Table 2. Model parameters

\begin{tabular}{l|l}
\hline$\pi$ & recruitment rate of external computers into the network \\
$\mu$ & disconnection rate of internal computers \\
$\beta_{2}$ & countermeasure propagation rate \\
$\beta_{1}$ & infection rate \\
$\sigma$ & infection rate suppressant which depends on countermeasure effectiveness \\
$\gamma_{1}$ & recovery rate of infectious computers \\
\hline
\end{tabular}

be the total number of internal computers at time $t$. Upon adding up the equations in (2.1) and in view of Eq. (3.1), we obtain

$$
\dot{N}=\pi-\mu N,
$$

which is a linear first order ordinary differential equation (ODE) with the property that

$$
\lim _{t \rightarrow \infty} N(t)=\frac{\pi}{\mu}
$$

The model (2.1) is well-posed in the practically feasible domain

$$
\mathbb{D}=\left\{(S, I, C) \in \mathbb{R}^{3} \mid S>0, I \geq 0, C \geq 0,0<N \leq \frac{\pi}{\mu}\right\} .
$$

Theorem 3.1. Suppose that $(S(0), I(0), C(0)) \in \mathbb{D}$, then system (2.1) has a unique solution that exists and remains in $\mathbb{D}$ for all $t \geq 0$.

Proof. The right hand side of system (2.1) is locally Lipschitz in $\mathbb{D}$, so a unique solution exists. Since $S+I+C=N \leq \frac{\pi}{\mu}$, we have $\dot{S}+\dot{I}+\dot{C} \leq 0$. According to (2.1) if $C=0$, then $\dot{C}=0$. Similarly, if $S=0$, then $\dot{S} \geq 0$. Moreover, according to the analytical solution of (3.2) and in view of equation (3.3), if $N(t)>0$ at $t=0$, then $N(t)>0$ for all $t>0$. We therefore conclude that the set $\mathbb{D}$ is positively invariant.

In order to simplify analysis of model (2.1), we consider the following equivalent system whereby $S$ is replaced by $N-I-C$ :

$$
\left\{\begin{aligned}
\dot{N} & =\pi-\mu N, \\
\dot{I} & =\beta_{1} I(N-I-C)-\beta_{2} C I-\left(\gamma_{1}+\mu\right) I+\sigma \beta_{1} C I, \\
\dot{C} & =\beta_{2} C I+\beta_{2} C(N-I-C)-\sigma \beta_{1} C I-\mu C .
\end{aligned}\right.
$$

\subsection{The disease-free equilibrium (DFE)}

The DFE point is a steady state solution of system (3.4) that is virus-free. Setting the right hand side of system (3.4) to zero, we obtain the following equilibria:

$$
\left\{\begin{aligned}
E_{1}=\left(N_{1}^{*}, I_{1}^{*}, C_{1}^{*}\right)=\left(\frac{\pi}{\mu}, 0,0\right), \\
E_{2}=\left(N_{2}^{*}, I_{2}^{*}, C_{2}^{*}\right)=\left(\frac{\pi}{\mu}, 0, \frac{\pi \beta_{2}-\mu^{2}}{\mu \beta_{2}}\right) .
\end{aligned}\right.
$$

The DFE point $E_{1}$ is of no interest to us because $C_{1}^{*}=0$. The point $E_{2}$ is the DFE of interest and only exists (meaning that it is nonnegative) if

$$
\pi \beta_{2}>\mu^{2} .
$$

In the rest of this paper we assume that Eq. (3.6) holds. 


\subsection{The basic reproduction number $\left(R_{0}\right)$}

The basic reproduction number is defined in [4] as the average number of secondary infections arising from a single infectious individual during his or her infectious period in a population of susceptible. To calculate $R_{0}$, we use the next generation matrix method by [21]. We consider equations representing the rate of change of Infectious classes which is only $\dot{I}$ in system (3.4). We rewrite the equation for $\dot{I}$ as a difference of two components $\mathcal{F}(N, I, C)$ and $\mathcal{V}(I, C)$ as

$$
\dot{I}=\mathcal{F}(N, I, C)-\mathcal{V}(I, C),
$$

where

$$
\mathcal{F}(N, I, C)=\beta_{1} I(N-I-C)+\sigma \beta_{1} C I,
$$

is the rate of appearance of new infections in the Infectious class and

$$
\mathcal{V}(I, C)=\beta_{2} C I+\left(\gamma_{1}+\mu\right) I,
$$

is the transfer rate of computers out of the Infectious state. Then,

$$
R_{0}=\left.\frac{\frac{\partial \mathcal{F}}{\partial I}}{\frac{\partial \mathcal{V}}{\partial I}}\right|_{E_{2}}=\frac{\sigma \pi \beta_{1} \beta_{2}+(1-\sigma) \beta_{1} \mu^{2}}{\pi \beta_{2}^{2}+\mu \gamma_{1} \beta_{2}} .
$$

\subsection{Local stability analysis of the DFE}

We linearise system (3.4) at the generalised DFE : $\left(N^{*}, 0, C^{*}\right)$ to obtain the Jacobian matrix

$$
J=\left(\begin{array}{ccc}
-\mu & 0 & 0 \\
0 & \sigma \beta_{1} C^{*}-\beta_{1} C^{*}+\beta_{1} N^{*}-\beta_{2} C^{*}-\left(\gamma_{1}+\mu\right) & 0 \\
\beta_{2} C^{*} & -\sigma \beta_{1} C^{*} & -\beta_{2} C^{*}
\end{array}\right) .
$$

In order to determine the local stability of $E_{1}$, we evaluate $J$ at $E_{1}$ to obtain the matrix $J_{1}$ with the following eigenvalues

$$
\lambda_{1}=-\mu, \quad \lambda_{2}=\frac{\pi \beta_{2}-\mu^{2}}{\mu}, \quad \lambda_{3}=\frac{\pi \beta_{1}-\mu \gamma_{1}-\mu^{2}}{\mu} .
$$

Since $\lambda_{2}>0$ by the assumption of Eq. (3.6), $E_{1}$ is unstable.

Similarly, for $E_{2}$, we obtain the matrix $J_{2}$ whose eigenvalues are

$$
\lambda_{1}=-\mu, \quad \lambda_{2}=-\left(\frac{\pi \beta_{2}-\mu^{2}}{\mu}\right), \quad \lambda_{3}=\frac{\left(\mu \beta_{2} \gamma_{1}+\pi \beta_{2}^{2}\right)\left(R_{0}-1\right)}{\mu \beta_{2}} .
$$

Theorem 3.2. The DFE $E_{2}$ is locally asymptotically stable $(L A S)$ if $R_{0}<1$ and unstable if $R_{0}>1$.

Remark 3.3. If $R_{0}=1$, then $\lambda_{3}=0$. This is a bifurcation point because there is a qualitative change of $E_{2}$ from being $L A S$ to unstable and vice-versa.

\subsection{The endemic equilibrium}

A steady state solution of the system (3.4) whereby the virus persists in the network is called the endemic equilibrium. A direct calculation reveals that there are endemic equilibria given by

$$
\left\{\begin{array}{l}
E_{3}=\left(N_{3}^{*}, I_{3}^{*}, C_{3}^{*}\right)=\left(\frac{\pi}{\mu}, \frac{\left(\mu \gamma_{1} \beta_{2}+\beta_{2}^{2}\right)\left(1-R_{0}\right)}{\mu \beta_{1}(1-\sigma)\left(\sigma \beta_{1}-\beta_{2}\right)}, \frac{\pi \beta_{2}-\mu^{2}}{\mu \beta_{2}}\right), \\
E_{4}=\left(N_{4}^{*}, I_{4}^{*}, C_{4}^{*}\right)=\left(\frac{\pi}{\mu}, \frac{\pi \beta_{1}-\mu\left(\gamma_{1}+\mu\right)}{\mu \beta_{1}}, 0\right) .
\end{array}\right.
$$

Similar to the DFE case, $E_{4}$ is of no interest in this study because $C_{4}^{*}=0$. 
Theorem 3.4. If one of the following statements holds

(i) $R_{0}>1$ and $\sigma<\frac{\beta_{2}}{\beta_{1}}$,

(ii) $R_{0}<1$ and $\sigma>\frac{\beta_{2}}{\beta_{1}}$,

then all components of $E_{3}$ are positive.

It is quite clear from Theorem 3.4, that model (3.4) can undergo a subcritical bifurcation at $R_{0}=1$. In order to fully understand the dynamics in the neighbourhood of $R_{0}=1$, we investigate the bifurcation direction by applying the Centre Manifold Theory.

\subsection{Elementary bifurcation}

In this subsection we briefly introduce relevant bifurcation theory of continuous and discrete systems. Consider a dynamical system

$$
\dot{z}=H(z, \phi), \quad z \in \mathbb{R}^{n}, \phi \in \mathbb{R},
$$

depending on a parameter $\phi$, where $H$ is sufficiently smooth.

Definition 3.5. A point $E^{* *}=\left(z^{*}, \phi^{*}\right)$ such that

$$
H\left(E^{* *}\right)=0
$$

is called an equilibrium point of system (3.12).

Definition 3.6. A point $z^{*} \in \mathbb{R}^{n}$ is called a permanent equilibrium point if $E^{*}=\left(z^{*}, \phi\right)$ is an equilibrium point for all $\phi$.

Let $J_{H}=D_{z} H(z, \phi)$ be the Jacobian matrix of the right hand side of system (3.12). Furthermore, let $\lambda_{i}=\lambda_{i}(\phi)(i=1,2, \cdots, n)$ be parameter dependent eigenvalues of the matrix $J_{H}\left(E^{*}\right)$. As the parameter $\phi$ is varied, it may happen that at least one of the eigenvalues crosses the imaginary axis. The point $\phi=\phi_{c}$ at which the real part of the eigenvalue is equal to zero (i.e. $\operatorname{Re}\left(\lambda_{i}\left(\phi_{c}\right)\right)=0$ for some $i$ ) is called the critical value of the parameter. If this happens, then the equilibrium point $E^{* c}=\left(z^{*}, \phi_{c}\right)$ is nonhyperbolic.

Remark 3.7. If an equilibrium point is nonhyperbolic, then linearisation technique fails to give a conclusive result regarding local asymptotic stability of nonlinear dynamical systems; see for instance [5] and [22]. In this case, the alternative mathematical tool is the centre manifold theory (CMT). CMT involves use of appropriate transformation to express the space $\mathbb{R}^{n}$ as a direct sum of the centre subspace $\mathbb{R}^{c}$, stable subspace $\mathbb{R}^{s}$ and unstable subspace $\mathbb{R}^{u}$ as follows:

$$
\mathbb{R}^{n}=\mathbb{R}^{c} \oplus \mathbb{R}^{s} \oplus \mathbb{R}^{u} \text { where } n=c+s+u,
$$

and $c, s$ and $u$ are positive integers; see [11] and [22] for details.

This paper is concerned with the case where the matrix $J_{H}\left(E^{* c}\right)$ has exactly one zero eigenvalue and all other eigenvalues have negative real parts. To determine local asymptotic stability of such a nonhyperbolic equilibrium point we consider the following theorem:

Theorem 3.8. [22] There exists a smooth centre manifold for Eq. (3.12). The dynamics of Eq. (3.12) restricted to the centre manifold is, for $u$ sufficiently small, given by the following 1-dimensional ODE

$$
\left\{\begin{array}{l}
\dot{u}=f(u, h(u, \phi), \phi), \quad(u, h(u, \phi), \phi) \in \mathbb{R} \times \mathbb{R}^{n-1} \times \mathbb{R}, \\
\dot{\phi}=0 .
\end{array}\right.
$$


Remark 3.9. Theorem 3.8 implies that the dynamics of system $(3.12)$ near $(z, \phi)=$ $\left(z^{*}, \phi_{c}\right)$ are topologically equivalent to the dynamics of Eq. $(3.15)$ near $(u, \phi)=\left(u^{*}, \phi_{c}\right)$ where $u^{*}$ is a permanent equilibrium point of system (3.15).

In order to gain insight into the qualitative features of the equilibrium point $E^{* c}$, we consider the following two restrictions of system (3.15):

$$
\dot{u}=f\left(u, \phi_{c}\right) \text { on } \mathcal{B}_{\delta}\left(u^{*}\right)
$$

and

$$
\dot{u}=f(u, \phi) \text { on } \mathcal{B}_{\delta}\left(u^{*}\right) \times\left(\phi_{c}-\epsilon, \phi_{c}+\epsilon\right)=: \mathcal{U}_{\delta, \epsilon}\left(u^{*}, \phi_{c}\right) \text {. }
$$

Definition 3.10. [22] An equilibrium point $\left(u^{*}, \phi\right)$ is said to undergo a bifurcation at $\phi=\phi_{c}$ if there exist $\delta>0$ and $\epsilon>0$ such that the qualitative features of the equilibrium point $\left(u^{*}, \phi\right)$ of system $(3.17)$ are not the same as those of the equilibrium point $\left(u^{*}, \phi_{c}\right)$ of system (3.16).

Remark 3.11. In many applications of dynamical systems it happens that the asymptotic stability of an equilibrium point changes due to bifurcation, but the equilibrium solution remains; see for instance [8].

Definition 3.12. Let $u^{*} \geq 0$ be a permanent equilibrium point of system (3.15). If the following statements hold,

(i) $u^{*}=0$ is locally asymptotically stable for $\phi<\phi_{c}$ and unstable for $\phi>\phi_{c}$,

(ii) for $\phi>\phi_{c}$, there exists a curve $u=u(\phi)>0$ of locally asymptotically stable equilibrium points,

then the bifurcation at the equilibrium point $\left(u^{*}, \phi_{c}\right)$ is supercritical (forward).

Definition 3.13. If statement $(i)$ of Definition 3.12 holds and for $\phi<\phi_{c}$, there exists a curve $u=u(\phi)>0$ of unstable equilibrium points, then the bifurcation at the equilibrium point $\left(u^{*}, \phi_{c}\right)$ is subcritical (backward).

There are some similarities between the bifurcation theory of continuous and discrete dynamical systems. In what follows we briefly point out some important aspects of the discrete case. Consider a discrete dynamical system

$$
z_{n+1}=F\left(z_{n}, \phi\right), \quad z_{n} \in \mathbb{R}^{n}, \phi \in \mathbb{R},
$$

depending on a parameter $\phi$, and $F$ is sufficiently smooth.

Definition 3.14. A point $\left(z^{*}, \phi^{*}\right)$ such that

$$
F\left(z^{*}, \phi^{*}\right)-z^{*}=0
$$

is called a fixed-point of system (3.18).

Remark 3.15. According to ([12, pp. 157]), "The bifurcation theory for fixed-points of system (3.18) with eigenvalue 1 is completely analogous to the bifurcation theory for equilibria of system (3.12) with eigenvalue $0 "$ ". Indeed if we let

$$
H\left(z_{n}, \phi\right)=F\left(z_{n}, \phi\right)-z_{n}
$$

then

$$
H\left(z^{*}, \phi\right)=0 \text { and } D_{z} H\left(z^{*}, \phi\right)=D_{z} F\left(z^{*}, \phi\right)-I_{n} .
$$

It is clear from Eq. (3.21) that $D_{z} H\left(z^{*}, \phi\right)$ will have a zero eigenvalue if $D_{z} F\left(z^{*}, \phi\right)$ has eigenvalue equal to one. 


\section{Bifurcation analysis}

\subsection{A continuous setting}

The analysis in this subsection is based on the result in [5] and here we only reproduce part of it for convenience. Consider the continuous system of ODEs

$$
\dot{x}=f(x, \phi),
$$

where the function $f: \mathbb{R}^{n} \times \mathbb{R} \rightarrow \mathbb{R}^{n}$ is sufficiently smooth and it is assumed $x=0$ is an equilibrium point of system (4.1) for all values of the parameter $\phi$, that is

$$
f(0, \phi) \equiv 0
$$

and

$$
A=D_{x} f(0,0),
$$

is the Jacobian matrix of the function $f(x, 0)$ at the point $x=0$.

Theorem 4.1. Assume the following:

A1: Zero is a simple eigenvalue of $A$ and all other eigenvalues of $A$ have negative real parts.

A2: Matrix A has a nonnegative right eigenvector $\mathbf{w}$ and a left eigenvector $\mathbf{v}$ corresponding to the zero eigenvalue such that $\mathbf{w}^{T} \cdot \mathbf{v}=1$.

Let $f_{k}$ be the $k^{\text {th }}$ component of $f$ and

$$
\left\{\begin{array}{l}
a=\sum_{k, i, j=1}^{n} v_{k} w_{i} w_{j} \frac{\partial^{2} f_{k}}{\partial x_{i} \partial x_{j}}(0,0) \\
b=\sum_{k, i=1}^{n} v_{k} w_{i} \frac{\partial^{2} f_{k}}{\partial x_{i} \partial \phi}(0,0)
\end{array}\right.
$$

where we assume that $b>0$. Then the local dynamics of system (4.1) around $x=0$ are determined by the sign of the number a as follows:

(i) If $a>0$, then the bifurcation at $\phi=0$ is subcritical.

(ii) If $a<0$, then the bifurcation at $\phi=0$ is supercritical.

Theorem 4.2. The model (3.4) undergoes a subcritical bifurcation at $R_{0}=1$ if $\sigma>$ $\frac{\mu}{\mu+\gamma_{1}}$.

Proof. Let

$$
\beta_{1}^{*}=\frac{\pi \beta_{2}+\gamma_{1} \mu \beta_{2}}{\sigma \pi \beta_{2}+(1-\sigma) \mu^{2}},
$$

and $\beta_{1}$ be the bifurcation parameter for model (3.4) such that if $\beta_{1}=\beta_{1}^{*}$, then $R_{0}=1$. Upon evaluating the Jacobian matrix $J$ in Eq. (3.8) at $\left(E_{2}, \beta_{1}^{*}\right)$ we obtain

$$
\left.J_{2}\right|_{\beta_{1}=\beta_{1}^{*}}=\left(\begin{array}{ccc}
-\mu & 0 & 0 \\
0 & 0 & 0 \\
\beta_{2} C^{*} & -\sigma \beta_{1}^{*} C^{*} & -\beta_{2} C^{*}
\end{array}\right),
$$

whose eigenvalues are

$$
\lambda_{1}=-\mu, \quad \lambda_{2}=-\left(\frac{\pi \beta_{2}-\mu^{2}}{\mu}\right), \quad \lambda_{3}=0 .
$$


The right eigenvector $\mathbf{w}$ associated with the zero eigenvalue $\lambda_{3}$ is given by

$$
\mathbf{w}=\left(0, \frac{-w_{3} \beta_{2}}{\sigma \beta_{1}^{*}}, w_{3}\right)^{T}, w_{3} \in \mathbb{R} .
$$

We choose $w_{3}=-1$, because $w_{2}$ corresponds to $I_{2}^{*}=0$ and thus has to be positive. Consequently,

$$
\mathbf{w}=\left(0, \frac{\beta_{2}}{\sigma \beta_{1}^{*}},-1\right)^{T} .
$$

The left eigenvector $\mathbf{v}$ associated with $\lambda_{3}=0$ is given by

$$
\mathbf{v}=\left(0, v_{2}, 0\right), \quad v_{2} \in \mathbb{R} .
$$

In order to fulfill the condition $\mathbf{w}^{\mathbf{T}} \cdot \mathbf{v}=\mathbf{1}$, we choose $v_{2}=\frac{1}{w_{2}}$ to obtain

$$
\mathbf{v}=\left(0, \frac{\sigma \beta_{1}^{*}}{\beta_{2}}, 0\right) \text {. }
$$

To avoid very messy calculations of the coefficients $a$ and $b$ in Eq. (4.4) and to be consistent with Theorem 4, we introduce the following notation

$$
\left\{\begin{aligned}
N & =x_{1}, I=x_{2}, C=x_{3}, \\
f_{1}\left(x_{1}, x_{2}, x_{3}\right) & =\dot{x}_{1}, \quad f_{2}\left(x_{1}, x_{2}, x_{3}\right)=\dot{x}_{2}, \quad f_{3}\left(x_{1}, x_{2}, x_{3}\right)=\dot{x}_{3} .
\end{aligned}\right.
$$

Since $v_{2}$ is the only non-zero component of $\mathbf{v}$, we only evaluate the second order partial derivatives of $f_{2}$ at $\bar{E}=\left(E_{2}, \beta_{1}=\beta_{1}^{*}\right)$ to obtain

$$
\left\{\begin{aligned}
\frac{\partial^{2} f_{2}}{\partial x_{1}{ }^{2}} & =0 ; \frac{\partial^{2} f_{2}}{\partial x_{1} \partial x_{2}}=\beta_{1}^{*} ; \frac{\partial^{2} f_{2}}{\partial x_{1} \partial x_{3}}=0 \\
\frac{\partial^{2} f_{2}}{\partial x_{3} \partial x_{2}} & =-(1-\sigma) \beta_{1}^{*}-\beta_{2} ; \frac{\partial^{2} f_{2}}{\partial x_{2}{ }^{2}}=-2 \beta_{1}^{*} ; \frac{\partial^{2} f_{2}}{\partial x_{3}{ }^{2}}=0 .
\end{aligned}\right.
$$

We consider equations (4.9), (4.11), and (4.13), in view of Eq. (4.4), to obtain

$$
a=\frac{2 \beta_{2}(1-\sigma) \mu\left(\gamma_{1}+\mu\right)}{\sigma^{2} \pi \beta_{2}+\sigma \mu^{2}(1-\sigma)}\left(\sigma-\frac{\mu}{\mu+\gamma_{1}}\right)
$$

and

$$
b=\frac{\sigma \pi \beta_{2}+\mu^{2}(1-\sigma)}{\mu \beta_{2}}>0 .
$$

The proof is complete.

\subsection{A discrete setting}

We construct a nonstandard finite difference (NSFD) scheme and prove that the scheme preserves the subcritical bifurcation property of the continuous model at $R_{0}=1$.

4.2.1. NSFD scheme. The introduction of the NSFD schemes goes back about three decades and is generally attributed to Mickens [17]. Many authors have, over the years, published extensively on the NSFD schemes with varying focus areas; see for instance $[1-3,9-11,14]$.

In general, the continuous system

$$
\frac{d z}{d t}=H(z), \quad z^{0}=z\left(t_{0}\right) \in \mathbb{R}^{n},
$$

where $H: U \subseteq \mathbb{R}^{n} \rightarrow \mathbb{R}^{n}$ is sufficiently smooth on a compact set $U$, cannot be completely solved by analytic techniques. Consequently, numerical methods are essential for gaining useful insights into the solution of such differential equations.

We consider a difference equation

$$
D_{\Delta t} z^{k}=G_{\Delta t}\left(H, z^{k}\right)
$$


which gives rise to a sequence $\left\{z^{k}\right\}_{k=0}^{\infty}$ of approximations to the solution $z(t)$ of (4.16) at the discrete time steps $\left\{t_{k}=t_{0}+k \Delta t\right\}_{k=0}^{\infty}$, where $\Delta t \equiv h$ is the step size,

$$
z\left(t_{k}\right) \approx z^{k},\left.D_{\Delta t} z^{k} \approx \frac{d z}{d t}\right|_{t=t_{k}},
$$

and $G_{\Delta t}\left(H, z^{k}\right)$ approximates the function $H(z)$ in system (4.16). It is worth noting that the algorithm in (4.17) enables one to find the discrete solution $z^{k+1}$ at the time $t_{k+1}$ whenever the solution $z^{k}$ is known at the time $t_{k}$.

Definition 4.3. ([1]) The difference equation in (4.17) is called a NSFD scheme if at least one of the following conditions is satisfied:

(i) In the first order discrete derivative $D_{\Delta t} z^{k}=\frac{z^{k+1}-z^{k}}{\Delta t}$, the classical denominator $h=\Delta t$ is replaced by a nonnegative function $\varphi:(0, \infty) \rightarrow(0, \infty)$ satisfying the asymptotic relation

$$
\varphi(\Delta t)=\Delta t+\mathcal{O}\left([\Delta t]^{2}\right)
$$

(ii) In the expression $G_{\Delta t}\left(H, z^{k}\right)$, some nonlinear terms are approximated in a nonlocal manner. For example, a term like $z^{2}\left(t_{k}\right)$ is approximated by $z^{k+1} z^{k}$ instead of $\left(z^{k}\right)^{2}$.

In this work however, we only construct a NSFD scheme that preserves positivity, elementary stability, and the bifurcation property at $R_{0}=1$. Having these dynamics in mind, we propose for system (3.4), the following NSFD scheme

$$
\left\{\begin{aligned}
\frac{N^{n+1}-N^{n}}{\varphi} & =\pi-\mu N^{n}, \\
\frac{I^{n+1}-I^{n}}{\varphi} & =\beta_{1} I^{n}\left(N^{n+1}-I^{n+1}-C^{n+1}\right)-\beta_{2} C^{n+1} I^{n}-\left(\gamma_{1}+\mu\right) I^{n}+\sigma \beta_{1} C^{n+1} I^{n}, \\
\frac{C^{n+1}-C^{n}}{\varphi} & =\beta_{2} C^{n+1} I^{n}+\beta_{2} C^{n}\left(N^{n}-I^{n}-C^{n}\right)-\sigma \beta_{1} C^{n+1} I^{n}-\mu C^{n},
\end{aligned}\right.
$$

where $\varphi=\varphi(\Delta t)=\Delta t+\mathcal{O}\left(\Delta t^{2}\right)$ as $\Delta t \longrightarrow 0$; see [17].

The scheme (4.20) may be expressed explicitly in the form

$$
\left\{\begin{aligned}
N^{n+1} & =\varphi \pi+(1-\varphi \mu) N^{n} \\
I^{n+1} & =\frac{\varphi C^{n+1} I^{n}\left(\sigma \beta_{1}-\beta_{2}-\beta_{1}\right)+\varphi \beta_{1} I^{n} N^{n+1}+\left[1-\varphi\left(\gamma_{1}+\mu\right)\right] I^{n}}{1+\varphi \beta_{1} I^{n}} \\
C^{n+1} & =\frac{\varphi \beta_{2} C^{n}\left(N^{n}-I^{n}-C^{n}\right)+(1-\varphi \mu) C^{n}}{1+\varphi\left(\sigma \beta_{1}-\beta_{2}\right) I^{n}}
\end{aligned}\right.
$$

For our purpose, we introduce the following denominator function

$$
\varphi=\frac{1-e^{-q \Delta t}}{q}, \quad q>\max S_{1} \bigcup S_{2}
$$

where

$$
\begin{gathered}
S_{1}=\left\{\gamma_{1}+\mu, \mu+N^{*}\right\}, S_{2}=\left\{\frac{\left|\lambda_{i}\right|^{2}}{2\left|\operatorname{Re} \lambda_{i}\right|}: i=1,2,3 \text { and } \operatorname{Re} \lambda_{i} \neq 0\right\}, \\
\left\{\lambda_{i}:\left|J_{2}-\lambda_{i} I\right|=0, \quad i=1,2,3\right\},
\end{gathered}
$$

$N^{*}$ is the upper bound of the total population, $I$ is the $3 \times 3$ Identity matrix and $J_{2}=\left.J\right|_{E_{2}}$.

Remark 4.4. It is worth mentioning that in equation (4.22), the terms $\left(\gamma_{1}+\mu\right)$ and $\left(\mu+N^{*}\right)$ guarantee nonnegativity of discrete solutions, while $\left(\max \frac{\left|\lambda_{i}\right|^{2}}{2\left|\operatorname{Re} \lambda_{i}\right|}: i=1,2,3\right)$ 
ensures that local asymptotic stability properties of hyperbolic equilibria of system (3.4) are preserved; see [11] and [14] for details.

4.2.2. Local stability analysis. Similar to the continuous setting, we linearise system (4.21) at the DFE: $\left(N^{*}, 0, C^{*}\right)$ to obtain the Jacobian matrix

$$
\begin{aligned}
J_{N S} & =\left(\begin{array}{ccc}
1-\varphi \mu & 0 & 0 \\
0 & 1+\varphi\left(\sigma \beta_{1} C^{*}-\beta_{1} C^{*}+\beta_{1} N^{*}-\beta_{2} C^{*}-\left(\gamma_{1}+\mu\right)\right) & 0 \\
\varphi \beta_{2} C^{*} & -\varphi \sigma \beta_{1} C^{*} & 1-\varphi \beta_{2} C^{*}
\end{array}\right) \\
& =I+\varphi J,
\end{aligned}
$$

where $I$ is the $3 \times 3$ Identity matrix and $J$ is defined in equation (3.8).

The eigenvalues of $J_{N S}$, denoted by $\lambda_{N S_{i}}$ are given by

$$
\lambda_{N S_{i}}=1+\varphi \lambda_{i}, \quad i=1,2,3,
$$

where $\lambda_{1}, \lambda_{2}, \lambda_{3}$ are the eigenvalues of the matrix $J$. A detailed account of the general schemes that are similar to scheme (4.21) can be found in [11].

Proposition 4.5. (i) If $\lambda_{i}<0$, then $\left|\lambda_{N S_{i}}\right|<1$.

(ii) If $\lambda_{i}>0$, then $\left|\lambda_{N S_{i}}\right|>1$.

(iii) If $\lambda_{i}=0$, then $\left|\lambda_{N S_{i}}\right|=1$.

Bifurcation analysis of scheme (4.21) relies on the results in [3] and [11]. For ease of reference, we reproduce it here. Consider a discrete dynamical system

$$
z_{n+1}=F\left(z_{n}, \phi\right), \quad z_{n} \in \mathbb{R}^{n}, \phi \in \mathbb{R},
$$

depending on a parameter $\phi, F$ is sufficiently smooth and $z^{*}$ is a fixed-point for all values of $\phi$.

Theorem 4.6. Assume that

(i) The Jacobian matrix $D=D_{z} F(0,0)$, has 1 as a simple eigenvalue, and all the other eigenvalues of $D$ have modulus less than 1.

(ii) The matrix $D$ has a nonnegative right eigenvector $\mathbf{w}$ and a left eigenvector $\mathbf{v}$ corresponding to the eigenvalue 1 such that $\mathbf{w}^{T} \cdot \mathbf{v}=1$.

Then the local dynamics of system (4.27) around the fixed-point $z^{*}=0$ are determined by the signs of the numbers $\tilde{a}$ and $\tilde{b}$ given below

$$
\begin{aligned}
\tilde{a} & =\sum_{k, i, j=1}^{n} v_{k} w_{i} w_{j} \frac{\partial^{2} F_{k}}{\partial z_{i} \partial z_{j}}(0,0), \\
\tilde{b} & =\sum_{k, i=1}^{n} v_{k} w_{i} \frac{\partial^{2} F_{k}}{\partial z_{i} \partial \phi}(0,0) .
\end{aligned}
$$

Assuming that $\tilde{b}>0$, then

(i) If $\tilde{a}>0$, then the bifurcation at $\phi=0$ is subcritical.

(ii) If $\tilde{a}<0$, then the bifurcation at $\phi=0$ is supercritical.

Theorem 4.7. If the continuous model (3.4) undergoes a subcritical bifurcation at $R_{0}=1$, so does scheme (4.21). 
Proof. Similar to the proof of Theorem 4.2, $\beta_{1}$ is a bifurcation parameter for scheme (4.20) such that if $\beta_{1}=\beta_{1}^{*}$, then $R_{0}=1$. Upon evaluating the Jacobian matrix $J_{N S}$ at $E^{*}=$ $\left(E_{2}, \beta_{1}=\beta_{1}^{*}\right)$ we obtain

$$
\left.J_{N S}\right|_{E^{*}}=\left(\begin{array}{ccc}
1-\varphi \mu & 0 & 0 \\
0 & 1 & 0 \\
\varphi \beta_{2} C^{*} & -\varphi \sigma \beta_{1}^{*} C^{*} & 1-\varphi \beta_{2} C^{*}
\end{array}\right),
$$

with eigenvalues

$$
\lambda_{1}=1-\varphi \mu, \quad \lambda_{2}=1-\varphi\left(\frac{\pi \beta_{2}-\mu^{2}}{\mu}\right), \quad \lambda_{3}=1 .
$$

The right and the left eigenvectors, $\mathbf{w}$ and $\mathbf{v}$ respectively, associated with $\lambda_{3}=1$ are the same as in the continuous setting. Similarly, we introduce the following notation

$$
\left\{\begin{aligned}
N^{n} & =z_{1}, I^{n}=z_{2}, C^{n}=z_{3}, \\
F_{1}\left(z_{1}, z_{2}, z_{3}\right) & =N^{n+1}, F_{2}\left(z_{1}, z_{2}, z_{3}\right)=I^{n+1}, F_{3}\left(z_{1}, z_{2}, z_{3}\right)=C^{n+1} .
\end{aligned}\right.
$$

We evaluate the second order partial derivatives of $F_{2}$ at $\bar{E}=\left(E_{2}, \beta_{1}=\beta_{1}^{*}\right)$ to obtain

$$
\left\{\begin{aligned}
\frac{\partial^{2} F_{2}}{\partial z_{1}{ }^{2}} & =0 ; \frac{\partial^{2} F_{2}}{\partial z_{3} \partial z_{2}}=\left(\varphi^{2} \beta_{2} C^{*}-\varphi\right)\left(\sigma \beta_{1}^{*}-\beta_{2}-\beta_{1}^{*}\right) ; \\
\frac{\partial^{2} F_{2}}{\partial z_{1} \partial z_{3}} & =0 ; \frac{\partial^{2} F_{2}}{\partial z_{3}^{2}}=0 ; \frac{\partial^{2} F_{2}}{\partial z_{1} \partial z_{2}}=\varphi^{2} \beta_{2} C^{*}\left(\sigma \beta_{1}^{*}-\beta_{2}-\beta_{1}^{*}\right), \\
\frac{\partial^{2} F_{2}}{\partial z_{2}{ }^{2}} & =-2 \varphi^{2} C^{*}\left(\sigma \beta_{1}^{*}-\beta_{2}-\beta_{1}^{*}\right) \sigma \beta_{1}^{*}-2 \varphi \beta_{1}^{*} .
\end{aligned}\right.
$$

We consider equations (4.9), (4.31), and (4.32), in view of Eq. (4.28), to obtain

$$
\begin{aligned}
\tilde{a} & =\varphi \frac{2 \beta_{2}(1-\sigma) \mu\left(\gamma_{1}+\mu\right)}{\sigma^{2} \pi \beta_{2}+\sigma \mu^{2}(1-\sigma)}\left(\sigma-\frac{\mu}{\mu+\gamma_{1}}\right) \\
& =\varphi a
\end{aligned}
$$

and

$$
\begin{aligned}
\tilde{b} & =\varphi \frac{\sigma \pi \beta_{2}+\mu^{2}(1-\sigma)}{\mu \beta_{2}}>0 \\
& =\varphi b,
\end{aligned}
$$

where $a$ and $b$ are defined in equations (4.14) and (4.15) respectively. The proof is complete.

\section{Numerical simulations and discussion}

We use numerical simulations to illustrate the existence of the supercritical and subcritical bifurcations at $R_{0}=1$. Furthermore, their impact on computer virus control strategies is discussed.

Example 5.1. We consider system (4.21) with parameters: $\gamma_{1}=0.02 ; \pi=1 ; \sigma=$ $0.02 ; \quad \beta_{2}=0.0015 ; \quad \mu=0.01 ; \quad \beta_{1}=0.1$.

$$
0.015=\frac{\beta_{2}}{\beta_{1}}<\sigma<\frac{\mu}{\gamma_{1}+\mu}=0.333 \text { and } R_{0}=5.0196>1 .
$$

In Fig. 3, we illustrate the supercritical bifurcation at $R_{0}=1$ of Fig. 2 by means of the NSFD (4.21). It is not surprising that the virus persists and it approaches a huge stable endemic equilibrium because $R_{0}=5.0196$ which is quite big. In an ideal situation, this means that a single infectious computer is capable of successfully infecting five more computers. In this case a simple intervention is to reduce $R_{0}$ to less than 1 by increasing $\beta_{2}$ (i.e. increasing the countermeasure propagation rate). 

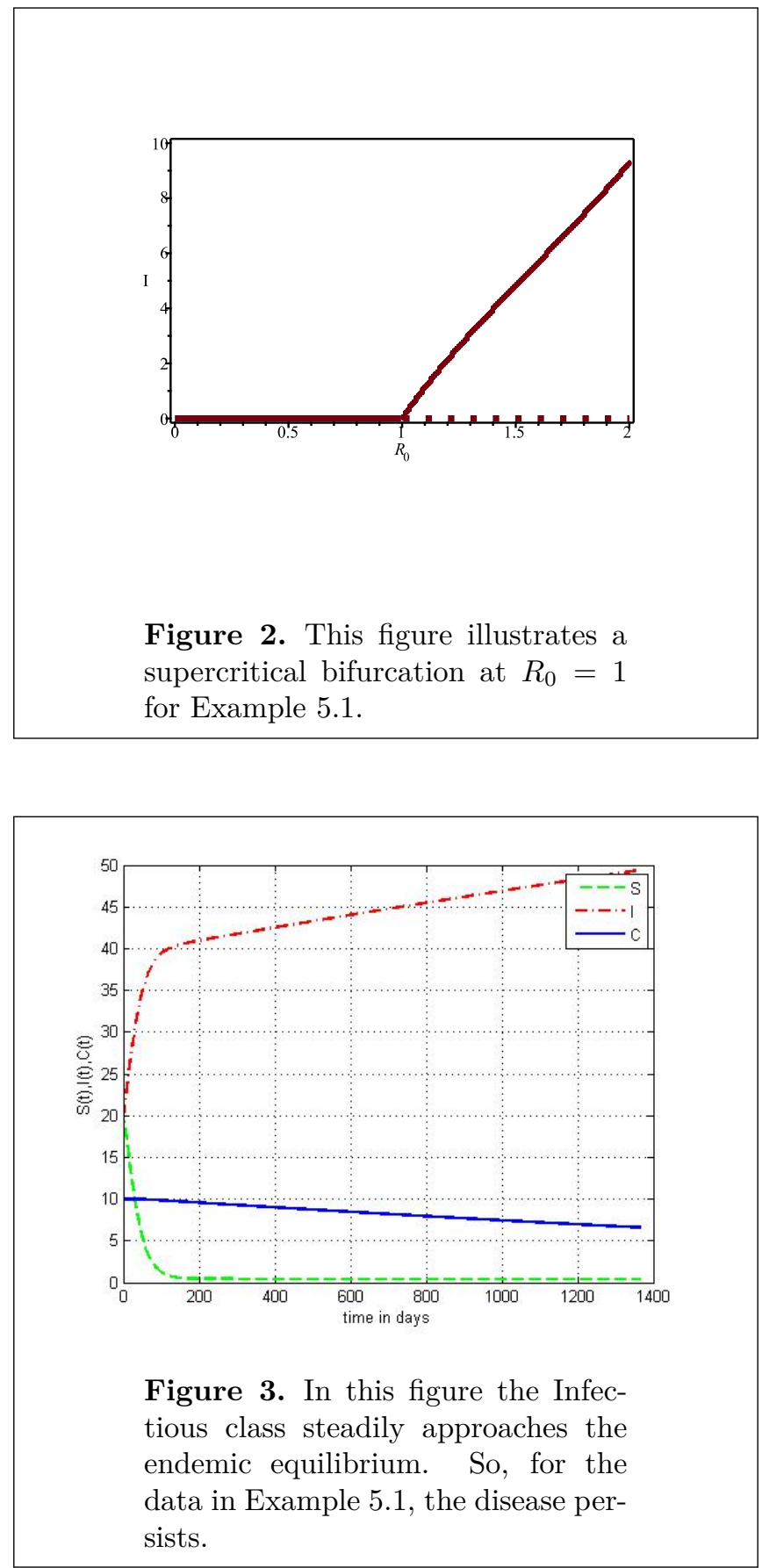

Example 5.2. We consider system (4.21) with parameters: $\gamma_{1}=0.7 ; \pi=1 ; \sigma=$ $0.15 ; \beta_{2}=0.01 ; \mu=0.01 ; \beta_{1}=0.08$.

$$
0.0141=\frac{\mu}{\gamma_{1}+\mu}<0.125=\frac{\beta_{2}}{\beta_{1}}<\sigma \text { and } R_{0}=0.7459<1 .
$$

In Fig. 5, we illustrate the subcritical bifurcation at $R_{0}=1$ of Fig. 4 . It is quite surprising that the disease persists and it approaches a huge stable endemic equilibrium even though $R_{0}<1$. In this case virus control is much harder because $R_{0}$ has to be decreased much more. 

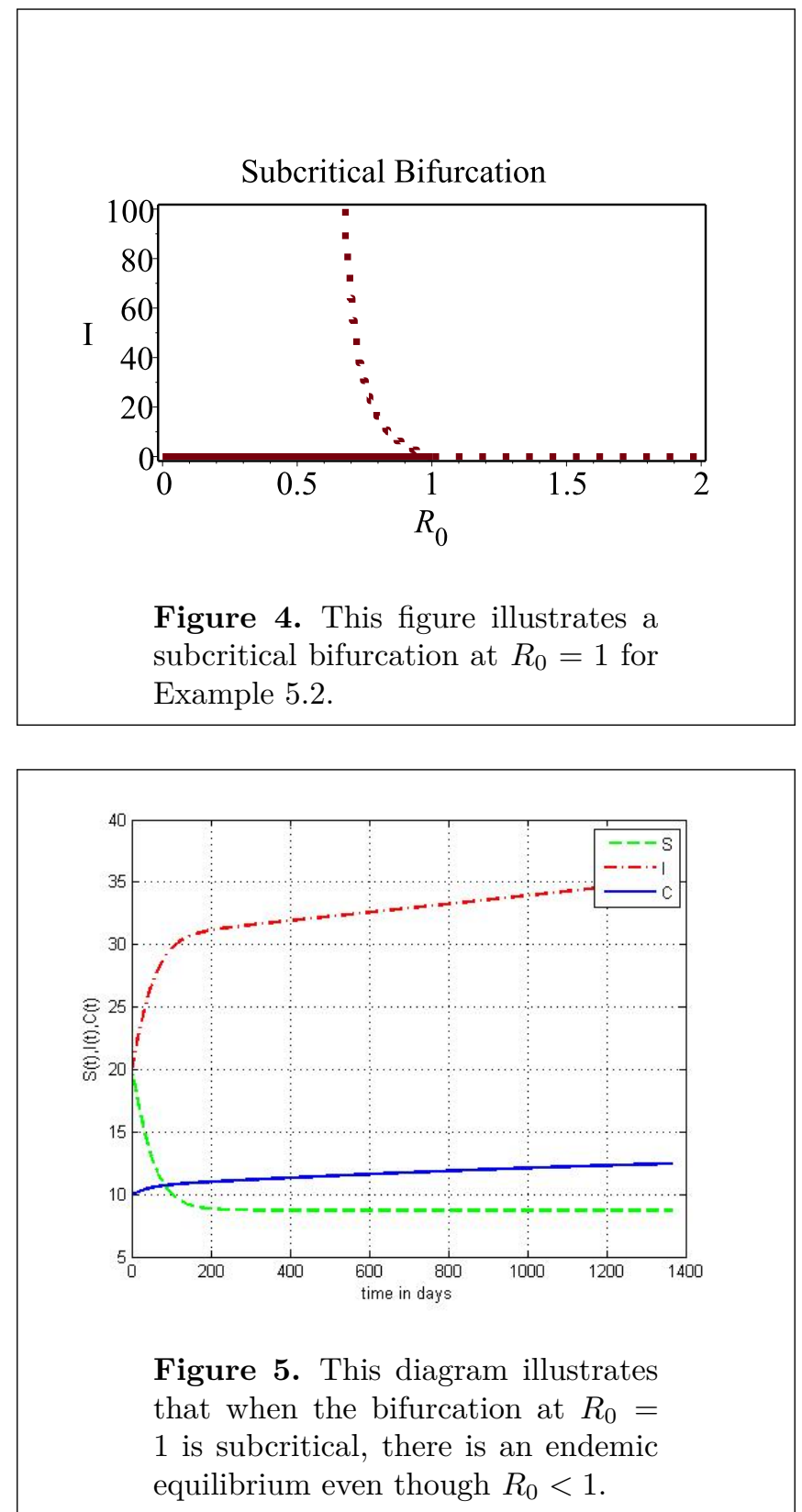

Example 5.3. We consider system (4.21) with parameters: $\gamma_{1}=0.7 ; \pi=1 ; \sigma=$ $0.019 ; \beta_{2}=0.01 ; \mu=0.01 ; \beta_{1}=0.08$.

$$
\frac{\mu}{\gamma_{1}+\mu}=0.0141<\sigma<0.125=\frac{\beta_{2}}{\beta_{1}} \text { and } R_{0}=0.1356<1 \text {. }
$$

In Fig. 7, we show that even though there is a subcritical bifurcation at $R_{0}=1$ as illustrated in Fig. 6, the virus can still be brought under control by decreasing $\sigma$ (i.e. by making sure that the control strategy is much more effective). It has to be noted that the virus propagation rate $\left(\beta_{1}\right)$ is larger than the countermeasure propagation rate $\left(\beta_{2}\right)$, but the virus can still be brought under control.

\section{Conclusion}

In this paper, we developed a new virus propagation mathematical model to study the impact of countermeasure competing (CMC) strategy on computer virus propagation 

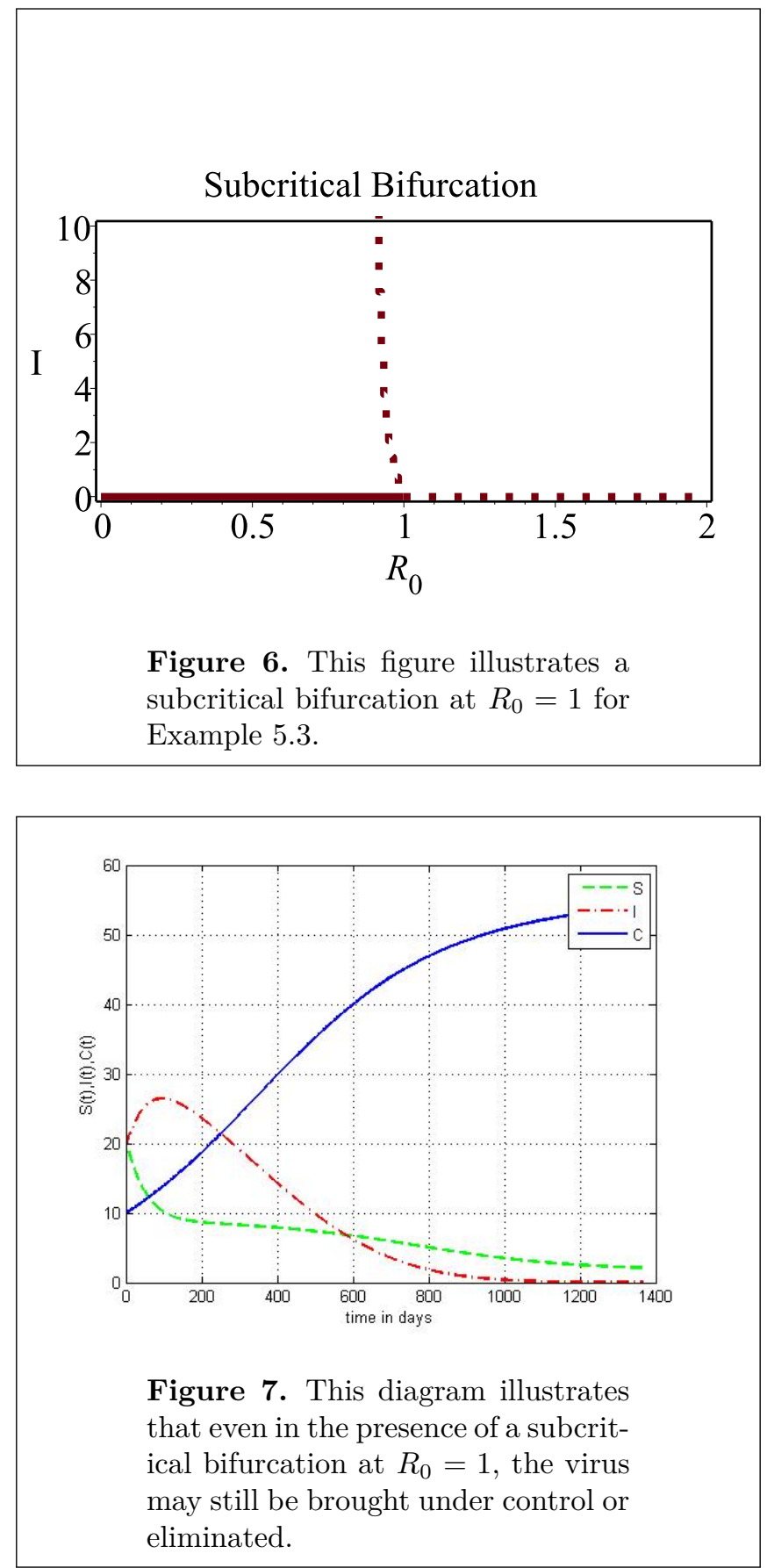

through information sharing network. A nonstandard finite difference (NSFD) scheme which preserves the bifurcation property of the continuous model at $R_{0}=1$ is constructed. Using the centre manifold theory, we established conditions under which the bifurcation is (i) supercritical and (ii) subcritical. We demonstrated numerically that, if a subcritical bifurcation occurs, reducing $R_{0}$ below 1 might not be sufficient to bring the virus under control.

The main conclusion of this study is that antivirus efficacy $(1-\sigma)$, virus propagation rate $\left(\beta_{1}\right)$ and countermeasure propagation rate $\left(\beta_{2}\right)$ are all essential for effective CMC strategy. More importantly, this study suggests that in cases where virus is propagated faster than the countermeasures, a very effective antivirus assists the CMC strategy in mitigating the impact of the virus on the network. 
Acknowledgment. First and foremost, the authors wish to extend deep gratitude to Prof. Emre Mengi for his detailed suggestions which have significantly improved the content of this manuscript. Heartfelt thanks is hereby extended to the anonymous reviewers for their meticulous work. Last but not least, the authors acknowledge the University of Pretoria, Department of Mathematics and Applied Mathematics for the provision of research facilities.

\section{References}

[1] R. Anguelov and J.M-S. Lubuma, Contributions to the mathematics of the nonstandard finite difference method and applications, Numer. Methods Partial Differential Equations, 17, 518-543, 2001.

[2] R. Anguelov and J.M-S. Lubuma, Nonstandard finite difference method by nonlocal approximation, Math. Comput. Simulation, 61 (3-6), 465-475, 2003.

[3] R. Anguelov, K. Dukuza and J.M-S. Lubuma, Backward bifurcation analysis for two continuous and discrete epidemiological models, Math. Methods Appl. Sci. 41 (18), 8784-8798, 2018.

[4] R.M. Anderson and R.M. May, Infectious Diseases of Humans: Dynamics and Control, Oxford University Press, USA, 1992.

[5] C. Castillo-Chavez and B. Song, Dynamical models of tuberculosis and their applications, Math. Biosci. Engin. 1, 361-404, 2004.

[6] L.C. Chen and K.M. Carley, The impact of countermeasure propagation on the prevalence of computer viruses, IEEE Trans. Syst., Man, Cybern. B. Cybern. 34 (2), 823-833, 2004.

[7] CSI/FBI; Computer crime and security survey. www.gocsi.com, 2008. Accessed 26 March 2020.

[8] J.D. Crawford, Introduction to bifurcation theory, Rev. Modern Phys. 63 (4), 991, 1991.

[9] Q.A. Dang and M.T. Hoang, Positivity and global stability preserving NSFD schemes for a mixing propagation model of computer viruses, J. Comput. Appl. Math. 374, $112753,2020$.

[10] D.T. Dimitrov and H.V. Kojouharov, Positive and elementary stable nonstandard numerical methods with applications to predator-prey models, J. Comput. Appl. Math. 189 (1-2), 98-108, 2006.

[11] N.K.K. Dukuza, Centre Manifold Theory for some Continuous and Discrete Epidemiological Models, University of Pretoria, PhD Thesis, South Africa, 2019.

[12] J. Guckenheimer and P. Holmes, Nonlinear oscillations, dynamical systems, and bifurcations of vector fields, 42, Springer Science \& Business Media, 2013.

[13] J.M. Heffernan, R.J. Smith and L.M. Wahl, Perspectives on the basic reproductive ratio, J. R. Soc. Interface, 2 (4), 281-293, 2005.

[14] P. Kama, Non-standard finite difference methods in dynamical systems, University of Pretoria, PhD Thesis, South Africa, 2009.

[15] W.O. Kermack and A.G. McKendrick, A contribution to the mathematical theory of epidemics, The Royal Society London, Proc. R. Soc. Lond. 115 (772), 700-721, 1927.

[16] W.O. Kermack and A.G. McKendrick, Contributions to the mathematical theory of epidemics. II.The problem of endemicity. The Royal Society London, Proc. R. Soc. Lond. 138 (834), 55-83, 1932.

[17] R. Mickens, Nonstandard Finite Difference Models of Differential Equations, World Scientific, Hackensack, NJ, 1994.

[18] B.K. Mishra and D.K. Saini, SEIRS epidemic model with delay for transmission of malicious objects in computer network, Appl. Math. Comput. 188 (2), 1476-1482, 2007. 
[19] W.H. Murray, The application of epidemiology to computer viruses, Comput. Secur. 7 (2), 130-150, 1988.

[20] J.R.C. Piqueira, A.A. de Vasconcelos, C.E.C.J. Gabriel and V.O. Araujo, Dynamic models for computer viruses, Comput. Secur. 27 (7-8), 355-359, 2008.

[21] P. Van den Driessche and J. Watmough, Reproduction numbers and sub-threshold endemic equilibria for compartmental models of disease transmission, Math. Biosci. 180 (1-2), 29-48, 2002.

[22] S. Wiggins, Introduction to Applied Nonlinear Dynamical Systems and Chaos, Springer-Verlag, Berlin, 1990.

[23] H. Yuan, G. Chen, J. Wu and H. Xiong, Towards controlling virus propagation in information systems with point-to-group information sharing, Decis. Support Syst. 48 (1), 57-68, 2009.

[24] Q. Zhu, X. Yang, L. Yang and X. Zhang, A mixing propagation model of computer viruses and countermeasures, Nonlinear Dynam. 73, 1433-1441, 2013. 